\title{
Strain-Specific Differences in Perinatal Rodent Oligodendrocyte Lineage Progression and Its Correlation with Human
}

\author{
Justin M. Dean ${ }^{a} \quad$ Matthew D. Moravec ${ }^{a} \quad$ Marjorie Grafe $^{b} \quad$ Nicholas Abend $^{e}$ \\ Jennifer Ren ${ }^{a} \quad X_{i}$ Gong $^{a} \quad$ Joseph J. Volpe ${ }^{f}$ Frances E. Jensen ${ }^{f} \quad$ A. Roger Hohimer ${ }^{c}$ \\ Stephen A. Back ${ }^{a}$ d \\ Departments of a Pediatrics, ${ }^{b}$ Pathology, ${ }^{c}$ Obstetrics and Gynecology and ${ }^{\mathrm{d}}$ Neurology, Oregon Health and Science \\ University, Portland, Oreg., ' Department of Neurology, Children's Hospital of Philadelphia, Philadelphia, Pa., and \\ fDepartment of Neurology, Children's Hospital and Harvard Medical School, Boston, Mass., USA
}

\section{Key Words}

Oligodendrocytes $\cdot$ Glia $\cdot$ White matter

\begin{abstract}
Progress in the development of rat models of human periventricular white matter injury (WMI) has been hampered by uncertainty about the developmental window in different rodent strains that coincides with cerebral white matter development in human premature infants. To define strainspecific differences in rat cerebral white matter maturation, we analyzed oligodendrocyte (OL) lineage maturation between postnatal days (P)2 and P14 in three widely studied strains of rat: Sprague-Dawley, Long-Evans and Wistar (W). We previously reported that late OL progenitors (preOL) are the major vulnerable cell type in human periventricular WMI. Strain-specific differences in preOL maturation were found at $P 2$, such that the $W$ rat had the highest percentage and density of preOL relative to the other strains. Overall, at P2, the state of OL maturation was similar to preterm human cerebral white matter. However, by P5, all three strains displayed a similar magnitude and extent of $\mathrm{OL}$ maturation that persisted with progressive myelination between P7 and P14. PreOL were the predominant $\mathrm{OL}$ lineage stage present in the cerebral cortex through $\mathrm{P} 14$, and thus $\mathrm{OL}$ lineage maturation
\end{abstract}

\section{KARGER}

(๑) 2011 S. Karger AG, Basel

Fax +4161306 1234

E-Mail karger@karger.ch

www.karger.com
Accessible online at: www.karger.com/dne occurred latter than in white matter. The hippocampus also displayed a later onset of preOL maturation in all three strains, such that OL lineage maturation and early myelination was not observed to occur until about P14. This timing of preOL maturation in rat cortical gray matter coincided with a similar timing in human cerebral cortex, where preOL also predominated until at least 8 months after full-term birth. These studies support that strain-specific differences in OL lineage immaturity were present in the early perinatal period at about $\mathrm{P} 2$, and they define a narrow window of preterm equivalence with human that diminishes by P5. Later developmental onset of preOL maturation in both cerebral cortex and hippocampus coincides with an extended window of potential vulnerability of the OL lineage to hypoxiaischemia in these gray matter regions.

Copyright $\odot 2011$ S. Karger AG, Basel

\section{Introduction}

White matter injury (WMI) to the developing cerebrum comprises a spectrum of lesions that predominate in survivors of premature birth and contribute to life-long spastic motor deficits from cerebral palsy as well as cognitive and learning disabilities [Volpe, 2009]. During human 
brain development, WMI peaks in incidence around 2832 weeks postconceptional age, has a predilection for periventricular white matter and topographically coincides with regions enriched in late oligodendrocyte $(\mathrm{OL})$ progenitors (preOL) [Haynes et al., 2003; Back et al., 2005; Talos et al., 2006a, b]. PreOL are selectively vulnerable to a spectrum of necrosis and apoptosis via mechanisms that include maturation-dependent susceptibility to oxidative stress, excitotoxicity and inflammatory cytokines [Jensen, 2005; Back, 2006; Volpe, 2008]. The spectrum of acute pathology includes macroscopic cystic or microscopic noncystic necrotic lesions (periventricular leukomalacia) with pancellular degeneration and more diffuse injury that selectively triggers OL lineage degeneration and later disturbances in myelination [Kinney and Armstrong, 2002].

Analysis of rat models of developmental WMI has been hampered in part by confusion regarding data from different strains of rat. During human brain development, preOL in cerebral white matter are selectively enriched throughout the high-risk period for WMI [Back et al., 2001; Jakovcevski and Zecevic, 2005]. However, the timing of appearance and distribution of preOL relative to later OL stages has not been systematically compared in different strains of rat in either white or gray matter. Thus, we quantified OL lineage maturation in three strains of rat that we and others have studied to define mechanisms of developing cerebral WMI: Sprague-Dawley (SD) [Fan et al., 2008; Segovia et al., 2008], Long-Evans (LE) [Follett et al., 2004; Manning et al., 2008] and Wistar (W) [Fontaine et al., 2008]. We report distinct strain-related differences in OL lineage maturation that may facilitate future studies relevant to the pathogenesis of WMI in the preterm newborn. We also identify that both the early postnatal rat and human infants display a similar immature pattern of OL lineage maturation in cerebral cortical gray matter.

\section{Materials and Methods}

Animals

Timed pregnant litters (culled to 10 pups per litter) of SD, LE and $\mathrm{W}$ rats were obtained from Charles River Laboratories (Wilmington, Mass., USA). At birth (postnatal day 0, P0), 20 animals from a minimum of 2 litters, delivered within $18 \mathrm{~h}$ of each other, were randomized. For each strain, brains were collected at P2 $(n=5), P 3(n=3)$, P5 $(n=5), P 7(n=5)$ and P14 $(n=2)$. There were no significant differences in animal weight among the three strains at each of the ages studied.

Immunohistochemical Methods and Quantification of O4- and O1-Labeled Cells

For each animal, cell counts were obtained from a minimum of three 50 - $\mu \mathrm{m}$-thick adjacent sections double-stained with a bio- tinylated $\mathrm{O} 4$ (bO4) and $\mathrm{O} 1$ monoclonal antibody $(1: 1,000)$ that were visualized with a $\mu$-chain-specific fluorescein-conjugated IgM secondary antibody (1:100; FI-2020, Vector Laboratories, Burlingame, Calif., USA) as previously described [Back et al., 2001]. Mature OL were visualized with the anti-myelin basic protein (MBP) antibody (SMI-99; 1:1,000; Covance, Berkley, Calif., USA). The nucleus was selected as the smallest countable object and was visualized by immunofluorescent counterstain with Hoechst 33342. Cell profiles that contained a nucleus were counted with a $\times 40$ objective equipped with a counting grid that was mounted on a Leica DMRA upright fluorescent microscope. At P2, P3, P5 and P7, cells were systematically counted in a minimum of 10 fields in a region that included the corpus callosum, the overlying supracallosal radiation and the external capsule. For comparative analysis of OL development in gray matter structures, we also examined preOL and immature OL maturation in the cerebral cortex at the levels of the midseptal nuclei and the anterior hippocampal formation/anterior nuclei of the thalamus.

Since the $\mathrm{O} 4$ antibody labels the entire population of preOL and immature OL, the total population of preOL and immature OL was defined by the number of O4-labeled cells. Immature OL were defined as the number of cells that labeled with the $\mathrm{O} 1$ antibody. PreOL were calculated as the difference between total (i.e. O4-labeled) cells and O1-labeled cells.

\section{Human Studies}

A series of 5 human cases was analyzed for OL lineage maturation in a standard region of parietal cortex collected at the trigone of the lateral ventricle. These cases were either previously reported by us [Back et al., 2001] or were subsequently collected prospectively from the pediatric autopsy population of the Department of Pathology at Oregon Health and Science University. Tissue was immersed at the time of collection in ice-cold $4 \%$ paraformaldehyde in $0.1 \mathrm{M}$ phosphate buffer and stored at $2-4^{\circ} \mathrm{C}$ until the time of analysis, as previously described [Back et al., 2001]. PreOL and immature OL were visualized in tissue sections processed and stained as described above for the rat studies. 'Normalcy' was defined as cases without gross brain pathology and was confirmed by histological data and review of the pertinent clinicopathological history. Only postmortem intervals of less than $24 \mathrm{~h}$ were accepted. All cases underwent brain autopsy by review of tissue that was processed for hematoxylin and eosin to permit evaluation by standard histopathologic criteria.

\section{Statistical Analysis}

Data are indicated as means \pm standard deviations. Comparisons between multiple groups were determined by one-way ANOVA, and significant differences probed with a post hoc Tukey honestly significant difference test.

\section{Results}

\section{OL Lineage Maturation in Cerebral White Matter} from $L E, S D$ and $W$ Rat

Most previous studies of rat perinatal WMI analyzed animals that ranged in age between P1 and P7. During this developmental window, the white matter is com- 

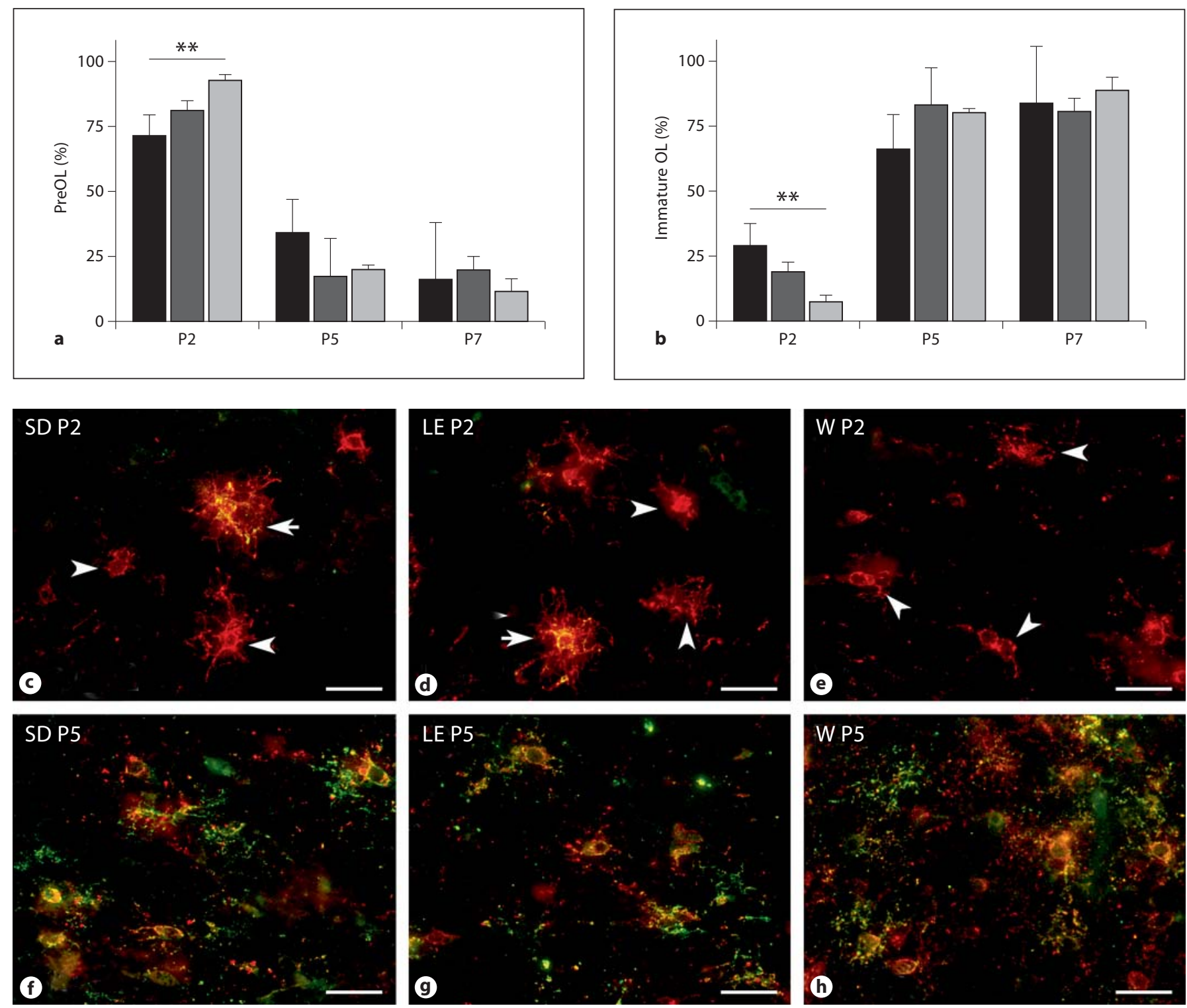

Fig. 1. OL lineage progression in the developing white matter of 3 rat strains. Percentage of preOL (a) and immature OL (b) in SD (black bars), LE (dark gray bars) and W rats (light gray bars) at P2, P5 and P7. ${ }^{* *} \mathrm{p}<0.01$ (ANOVA; Tukey's multiple comparison test). Representative photomicrographs at P2 (c-e) and P5 (f-h) of preOL (red; arrowheads) and immature OL (yellow; arrows) in corpus callosum double-labeled with bO4 (red) and O1 (green). Scale bars $=40 \mu \mathrm{m}$. c, $\mathbf{f}$ SD rat. $\mathbf{d}, \mathbf{g}$ LE rat. e, $\mathbf{h} \mathrm{W}$ rat. prised of OL progenitors, preOL and immature OL. Since we previously found that OL progenitors are very resistant to hypoxia-ischemia [Back et al., 2002], we focused our analysis of OL lineage maturation on preOL and immature OL in a region of particular predilection for cerebral injury, the subcortical white matter of the corpus callosum, the overlying supracallosal radiation and the external capsule. We determined the percentages of preOL (fig. 1a) and immature OL (fig. 1b) in SD, LE and W rats at P2, P5 and P7. In all three strains, preOL predominated at $\mathrm{P} 2$ and immature OL were a minor population. OL lineage maturation in the $\mathrm{W}$ rat was the least advanced, such that $92.5 \pm 2.4 \%$ of the total O4-labeled cells were preOL. By contrast, maturation was the most advanced in the SD rat at $\mathrm{P} 2$, such that $29.1 \pm 8.5 \%$ of the total $\mathrm{O} 4$ labeled cells were immature OL.

Despite these strain-specific differences in the rate of preOL maturation in the early perinatal period, all three 

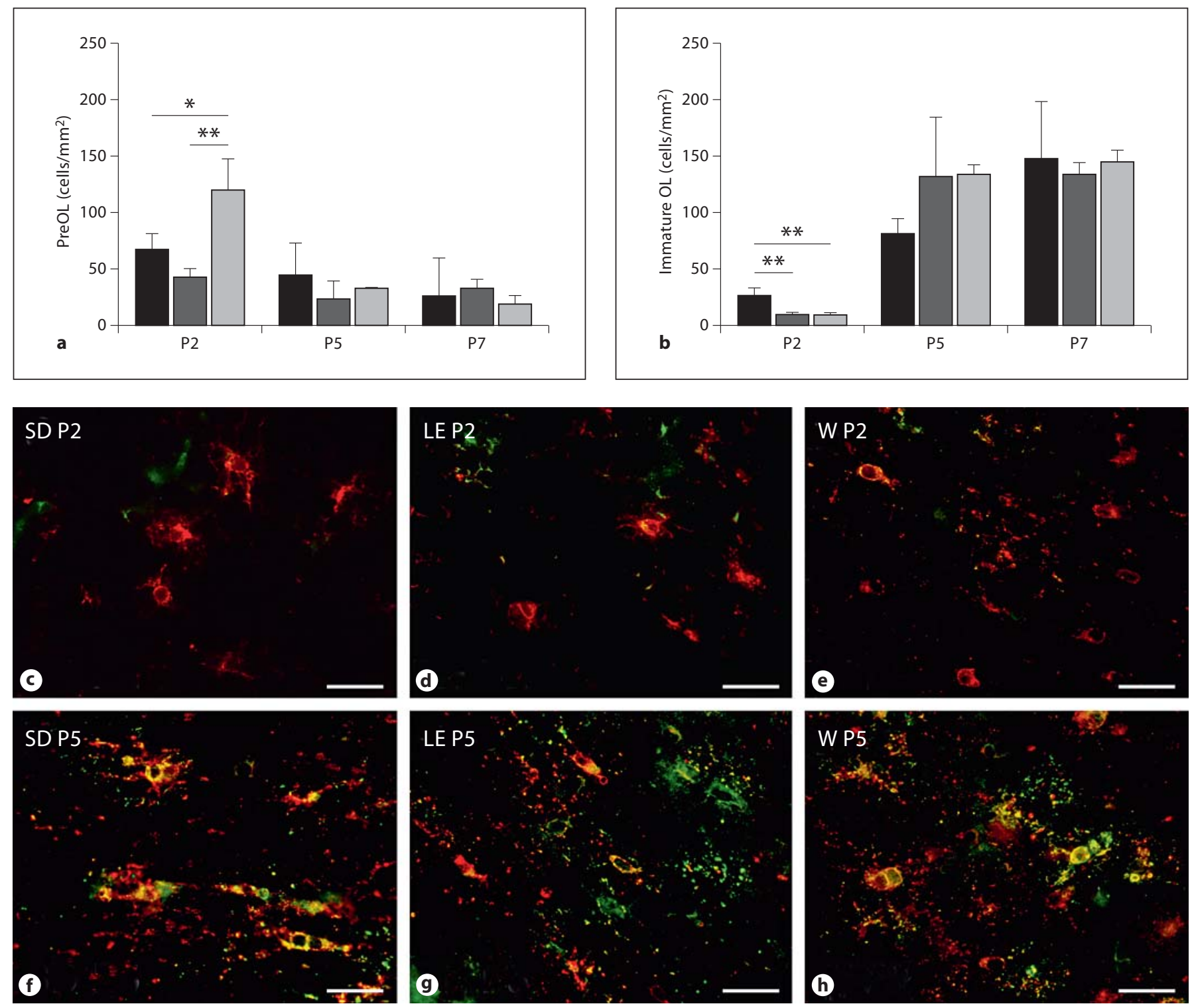

Fig. 2. Strain-specific differences in the density of preOL (a) and immature OL (b) in SD (black bars), LE (dark gray bars) and W rats (light gray bars) at $\mathrm{P} 2$ relative to $\mathrm{P} 5$ and $\mathrm{P} 7 .{ }^{*} \mathrm{p}<0.05 ;{ }^{* *} \mathrm{p}<$ 0.01 (ANOVA; post hoc Tukey multiple comparison test). Repre- sentative photomicrographs at P2 (c-e) and P5 (f-h) of preOL (red) and immature OL (yellow) in corpus callosum double-labeled with bO4 (red) and O1 (green). Scale bars $=40 \mu \mathrm{m}$. c, f SD rat. $\mathbf{d}, \mathbf{g} \mathrm{LE}$ rat. $\mathbf{e}, \mathbf{h} \mathrm{W}$ rat. strains displayed a similar rapid maturation at P5, such that preOL were a minor population and immature OL predominated. Consistent with this rapid progression of OL lineage maturation, all three strains contained more than $80 \%$ immature OL at P7 with no significant differences between strains. Thus, over a 5-day span of early white matter maturation, preOL rapidly transitioned from the major to the minor population of OL lineage cells.
Since we have found that the density of preOL in the $\mathrm{SD}$ rat contributes to the magnitude of WMI from hypoxia-ischemia between P2 and P7 [Back et al., 2002], we next determined whether strain-specific differences in preOL and immature OL densities might exist between P2 and P7. PreOL density at $\mathrm{P} 2$ was highest in $\mathrm{W}$ rats (fig. 2a) compared with the SD ( $p<0.05$; one-way ANOVA with Tukey's post hoc comparison) and the LE rats $(\mathrm{p}<0.01)$. 

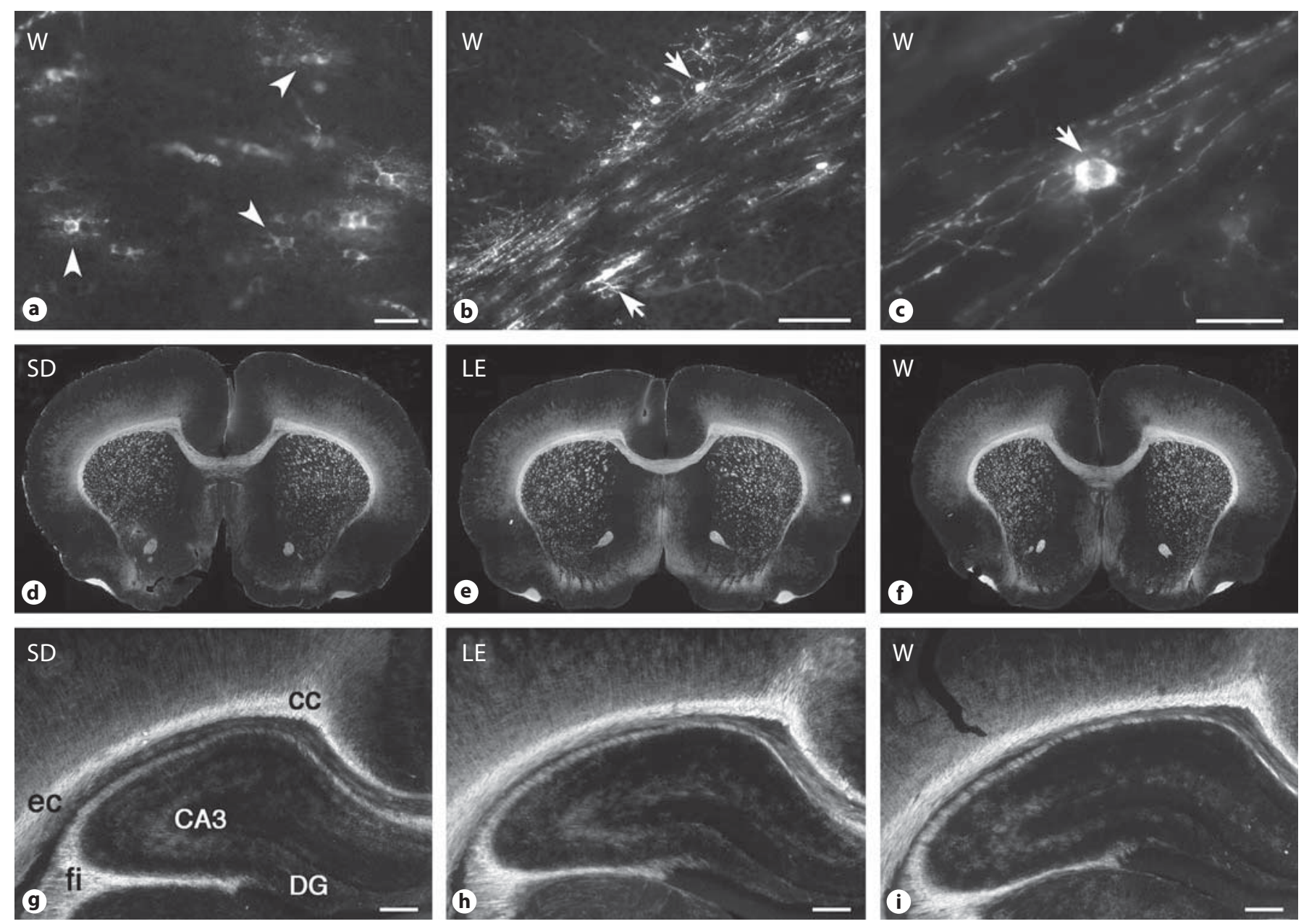

Fig. 3. Myelination pattern visualized by MBP staining in W (a-c, $\mathbf{f}, \mathbf{i}), \mathrm{SD}(\mathbf{d}, \mathbf{g})$ and LE rats $(\mathbf{e}, \mathbf{h})$. Shown are images at the midline corpus callosum (a) and external capsule (b, low power; c, high power) at P5, low-power coronal images at the level of the midseptal nuclei at P14 (d-f), and higher-power coronal images of the hippocampal formation and the adjacent white matter tracts of the corpus callosum (cc), external capsule (ec) and the fimbria of the fornix (fi) at P14 (g-i). Arrowheads: MBP-positive nonmyelinating OL. Arrows: MBP-positive myelinating OL. CA3 = Cornu ammonis area 3; DG $=$ dentate gryus. Scale bars $=50 \mu \mathrm{m}(\mathbf{a}, \mathbf{c})$, $200 \mu \mathrm{m}(\mathbf{b})$ and $500 \mu \mathrm{m}(\mathbf{g}-\mathbf{i})$.
However, with white matter maturation at P5 and P7, there was a similarly low density of preOL in all three strains. The density of immature OL at P2 was much lower than of preOL in all three strains (fig. 2b), and the immature OL density was highest in SD rats compared with $\mathrm{LE}$ and $\mathrm{W}$ rats $(\mathrm{p}<0.01)$. With white matter maturation at P5 and P7, there was a marked increase in the density of immature OL, with a similar density in all three strains.

Despite the similar maturation of immature OL at P5, there were notable differences in the terminal differentiation of mature OL at this age. Numerous MBP-positive mature OL somata were visualized in the midline corpus callosum of W rats (fig. 3a), whereas markedly fewer were visualized in SD and LE rats (not shown). Surprisingly, extensive myelination was also detected in the external capsule of $\mathrm{W}$ rats (fig. 3b, c), whereas very few weakly stained MBP-labeled somata were visualized in the SD and LE rats (not shown). At P14, there was a similar pattern of myelination seen in cerebral white matter structures among all the three strains (fig. $3 \mathrm{~d}-\mathrm{f}$ ).

\section{OL Lineage Maturation in Cerebral Gray Matter}

Although OL lineage maturation appears to occur later in gray matter relative to white matter, strain-related 


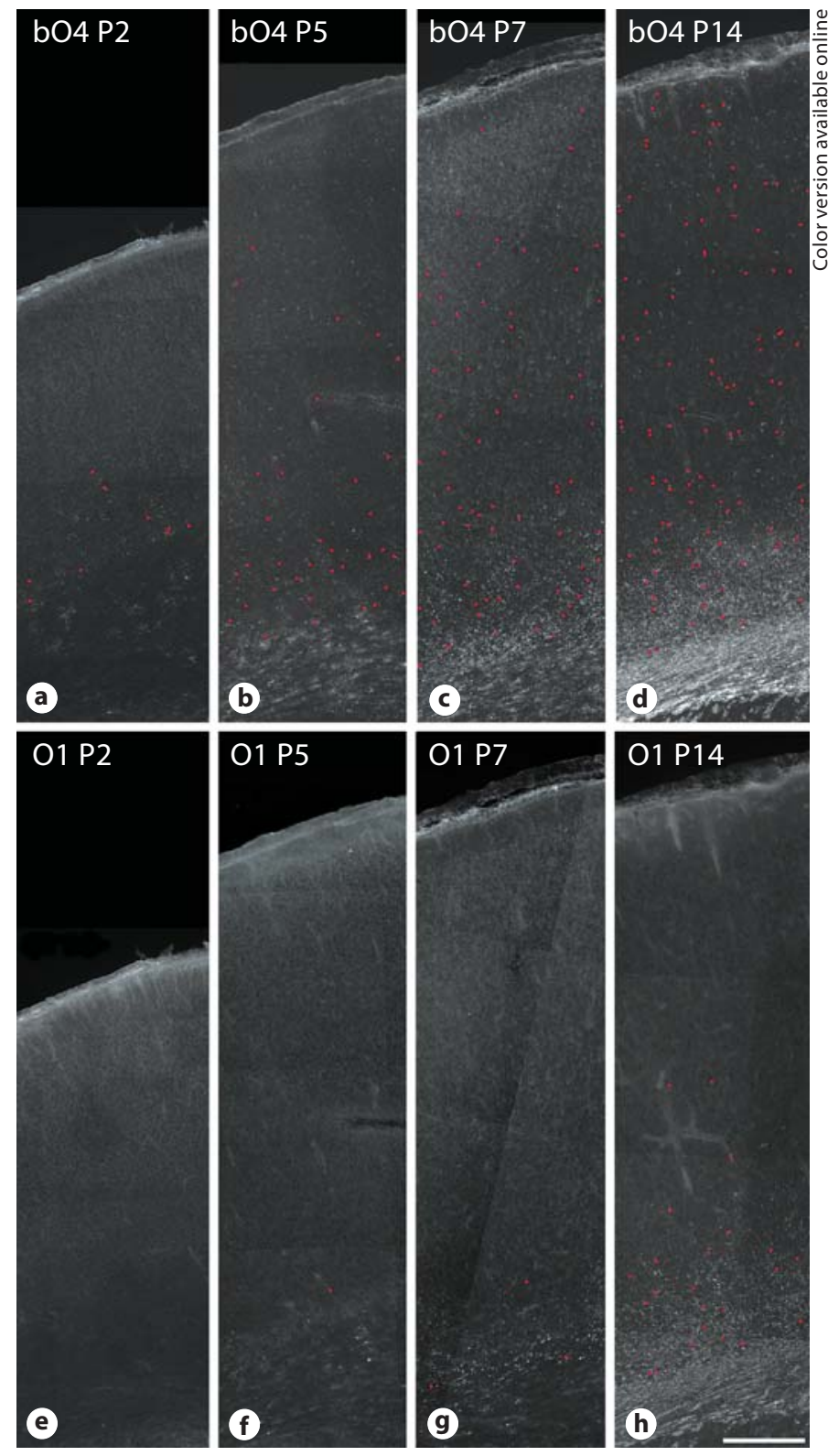

Fig. 4. OL lineage progression in the cerebral cortex between P2 and P14. Low-power photomicrographs of the distribution of cells stained for bO4 (a-d) and O1 (e-h) at P2 (a, e), P5 (b, f), P7 (c-g) and P14 (d, h) in the SD rat. Individual somata are indicated by the red circles (online version only). Note the progressive increase in density of bO4-labeled cells within the full thickness of the cerebral cortex by P14. Relatively few O1-labeled cells are seen in $\mathbf{e - h}$, supporting that the majority of the bO4-labeled cells in the cortex are preOL. Scale bar $=250 \mu \mathrm{m}$. differences have not been studied. We thus determined the relative maturation of preOL between $\mathrm{P} 2$ and P14 in two widely studied gray matter structures: the cerebral cortex and the hippocampus. In the SD rat cerebral cortex, between $\mathrm{P} 2$ and $\mathrm{P} 7$, the majority of cells were labeled with the bO4 antibody (fig. 4a-c), but O1-labeled immature OL were rarely seen (fig. $4 \mathrm{e}-\mathrm{g}$ ). This indicated that almost all bO4-labeled cortical cells were preOL. The timing of immature OL maturation was similar in the LE and $\mathrm{W}$ rats (data not shown). At P2, a low number of preOL were visualized predominantly in the lower half of the cortex. At P5, P7 and P14 there was a similar progressive expansion of preOL in the cortex, with preOL present within all cortical layers by P14 (fig. 4d). At P7, O1-labeled immature OL were rarely seen, except in deep cortical layers adjacent to the subcortical white matter. By P14, greater numbers of immature OL were present in the lower third of the cortex (fig. 4h) in a pattern similar to the advancement of myelination defined by MBP staining (fig. 3).

Within the hippocampus, OL maturation was markedly delayed at P7 relative to adjacent white matter tracts such as the corpus callosum, external capsule and fimbria of the fornix, where extensive early myelination was present (fig. 5a). For all three strains, low numbers of preOL were present in the hippocampus at P5 (not shown). By $\mathrm{P} 7$, preOL were visualized throughout the cornu ammonis area (CA)1-CA3 and within the dentate gyrus (fig. 5c), while immature OL were rarely seen (fig. 5e). By P14, robust myelination was present in the white matter tracts adjacent to the hippocampus, as visualized by $\mathrm{O} 4$ labeling (fig. 5b) and staining for MBP (fig. 3g-i). Within the hippocampus, however, lower levels of MBP expression (fig. $3 g-i)$ coincided with the presence of numerous immature OL that labeled with both the $\mathrm{O} 4$ (fig. 5d) and $\mathrm{O} 1$ (fig. 5f) antibodies. Myelination within the hippocampus was most pronounced in CA3, with lower levels within $\mathrm{CA} 1$ and the dentate gyrus. Hence, all three strains of rat showed a similar pattern of delayed maturation of the OL lineage in the hippocampus relative to adjacent white matter tracts.

\section{OL Lineage Maturation in Human Cerebral Cortex}

Given the consistent delay in preOL maturation in all three strains of rat at P14, we determined whether human cerebral cortex also displayed a similar later onset of preOL maturation relative to white matter. The parietal cortex and white matter were examined from 5 human brains that ranged in age from 37 postconceptional weeks to 8 months after full-term birth. Figure 6 demonstrates 

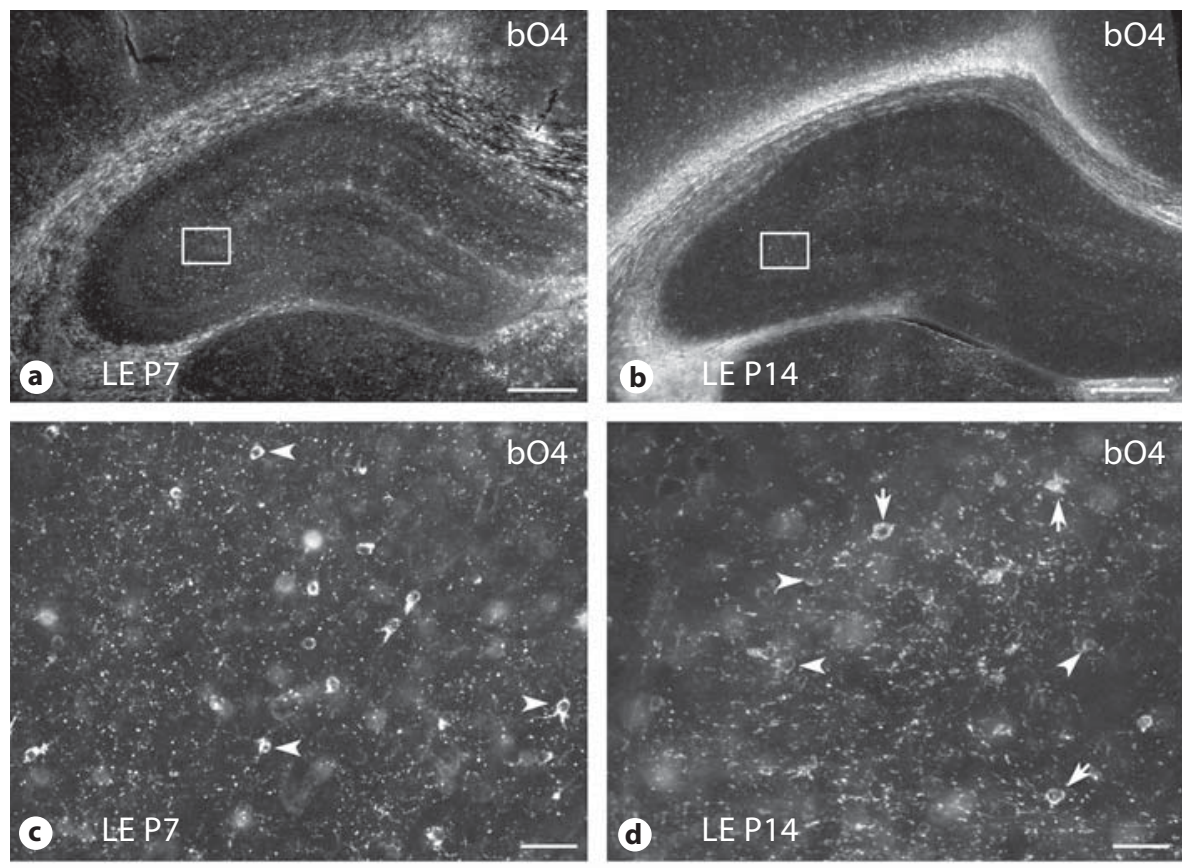

Fig. 5. OL lineage progression in the hippocampus. Low-power photomicrographs of the distribution of bO4-labeled cells and myelin at P7 (a) and P14 (b) in the LE rat. Higher-power images of double-immunohistochemical staining for bO4 (c, d) and $\mathrm{O} 1(\mathbf{e}, \mathbf{f})$ as visualized in CA3 (box) are shown for P7 (c, e) and P14 (d, f). PreOL (arrowheads) labeled for bO4 but not O1. Immature OL (arrows) labeled for bO4 and O1. Scale bars $=1.0 \mathrm{~mm}(\mathbf{a}, \mathbf{b})$ and 100 $\mu \mathrm{m}$ (c-f).
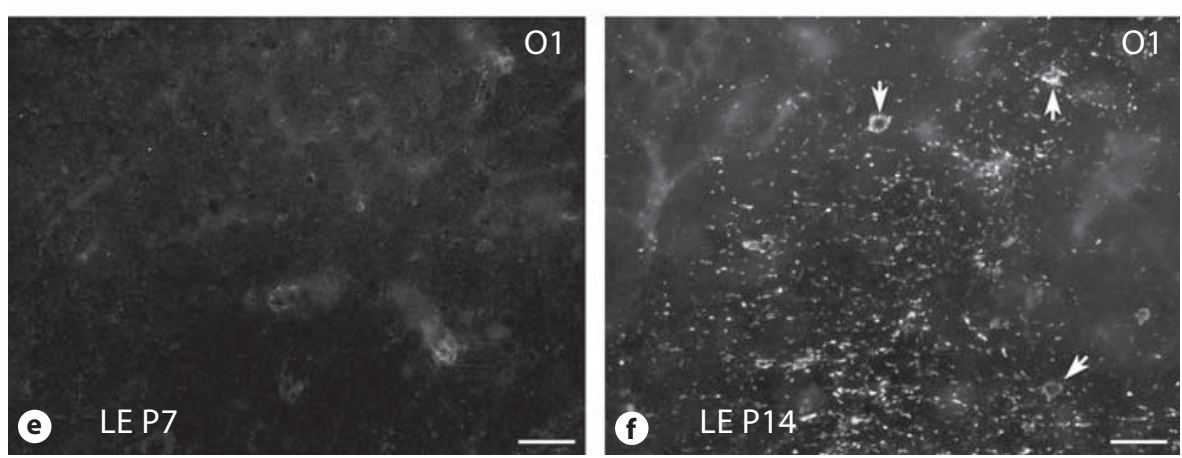

that human preOL predominated in cerebral cortex through 8 months. At 1 month after full-term birth (fig. 6a, c), cerebral cortex contained numerous preOL, despite the fact that early myelination was detected in adjacent subcortical white matter. Immature OL, labeled with the $\mathrm{O} 1$ antibody, were not observed (data not shown). At 8 months (fig. 6b, d), the subcortical white matter was heavily myelinated, but preOL were still visualized throughout the full thickness of the cortex. However, both preOL and immature OL were visualized in regions of early myelination at the boundary between cortical layer 6 and the subcortical white matter (fig. 6e). Hence, during infancy in the first year of life, OL lineage maturation was less pronounced in the parietal cortex relative to the white matter.

Strain-Dependent Differences in Rat OL Maturation

\section{Discussion}

Although perinatal rodents have significant limitations as neuropathological models of human WMI [Back et al., 2006], they have provided powerful access to questions related to cellular and molecular mechanisms of WMI. There is, thus, a critical need to validate existing rat models of WMI to identify the unique and common features provided by different strains of rat. Toward this end, we defined OL lineage maturation in three commonly studied strains of rat. We particularly focused on the human preterm-equivalent window in development when susceptible preOL predominated in each strain. Our major findings were the following: (1) for all three strains, preOL levels were highest in the white matter at $\mathrm{P} 2$, with preOL at the highest percentage in $\mathrm{W}$ compared 
Fig. 6. OL maturation is less pronounced in human parietal cortex compared with white matter. Low-power photomicrographs show numerous cells labeled with bO4 antibody at 1 month (a) and 8 months (b) after full-term birth. Note that the bO4-labeled cells in the cortex were preOL that did not stain for the $\mathrm{O} 1$ antibody (not shown). a, b The approximate boundary between cortical layer 6 and the subcortical white matter is indicated by the arrowheads and was confirmed by a Hoechst fluorescent counterstain (not shown). c, d Higher-power details of the preOL (arrowheads) from the cases in a (c) and $\mathbf{b}$ (d) demonstrate the simplified arbor of processes associated with the cortical preOL at both ages. e Both preOL (red, online version only; arrowheads) and immature OL (yellow, online version only; arrows) were visualized in a region of early myelination at the edge of the densely myelinated tract shown in $\mathbf{b}$. Scale bars $=200$ $\mu \mathrm{m}(\mathbf{a}, \mathbf{b})$ and $50 \mu \mathrm{m}(\mathbf{c}-\mathbf{e})$.
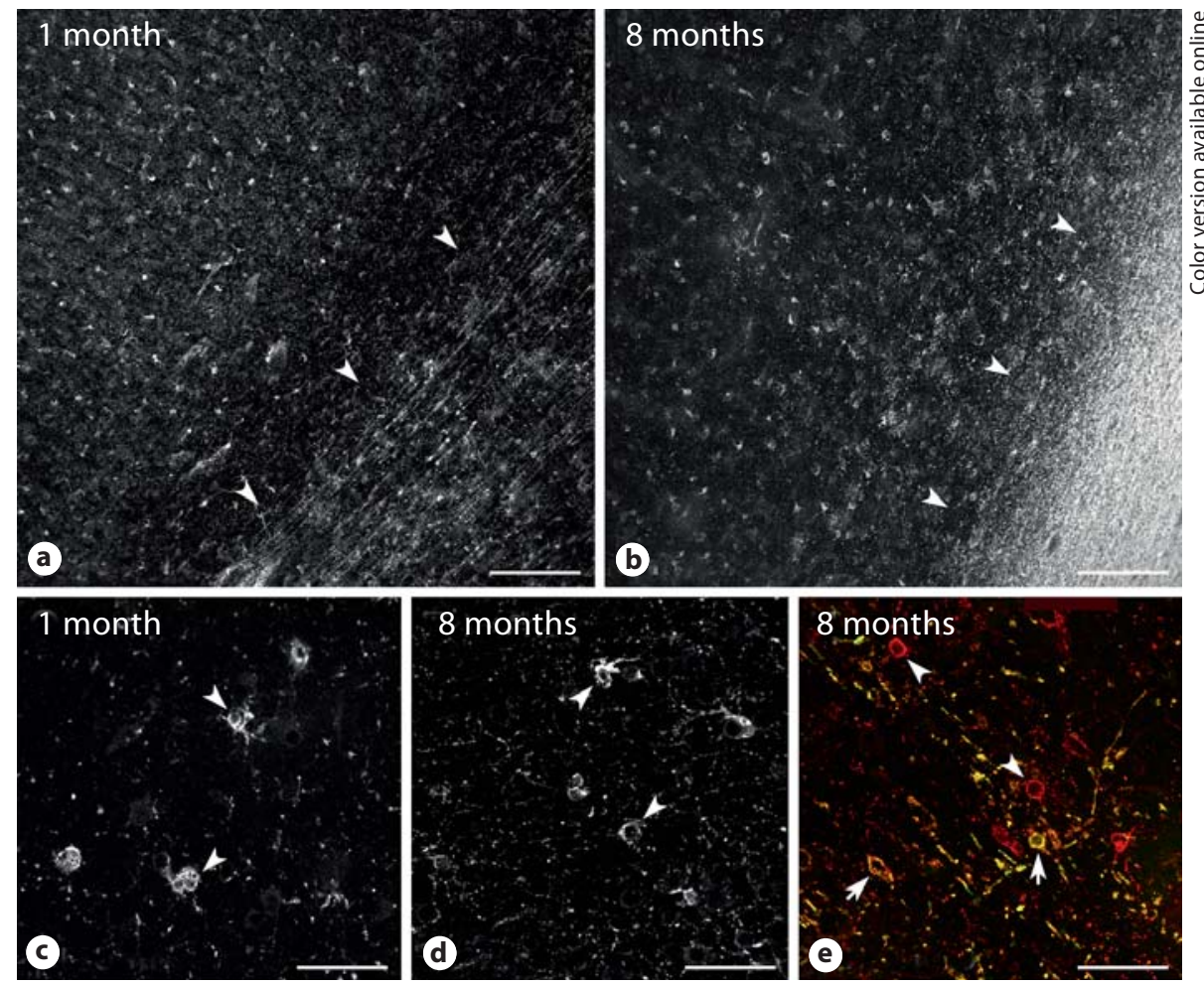

with SD and LE rats; (2) at P2, preOL density differed markedly among strains, with the highest found in W rats; (3) maturation of preOL to immature OL occurred rapidly in all three strains, such that by $\mathrm{P} 5$, preOL were a minor population in all three strains; (4) mature OL were increased and myelination was advanced at $\mathrm{P} 5$ in $\mathrm{W}$ relative to SD and LE rats; (5) no major differences in the distribution of myelination were observed among strains at P14, and (6) all three strains showed a similar later onset of OL lineage maturation in the cerebral cortex and hippocampus, such that preOL predominated despite the advance of myelination in adjacent white matter tracts. Of note, there were no obvious differences in size and morphology of preOL or OL between the different strains in either the white matter or the cortex.

In all three strains of rat there was a narrow developmental window in the perinatal period when preOL predominated in white matter. This difference in preOL density at $\mathrm{P} 2$ suggests that an equivalent hypoxic-ischemic insult could result in greater WMI in W rats because of the higher percentage of preOL and the higher preOL density at P2. By comparison, the SD rat at $\mathrm{P} 2$ had the lowest relative percentage of preOL and an intermediate preOL density. Nevertheless, in the SD rat, approximate- ly $70 \%$ of total preOL degenerated acutely following hypoxia-ischemia [Back et al., 2002]. Future studies are needed to determine whether differences in preOL density or proportion will translate into equivalent differences in susceptibility to hypoxia-ischemia. It is also possible that differences in a variety of intrinsic or extrinsic factors may influence preOL susceptibility in each strain of rat. Such intrinsic factors might include strain-related differences in antioxidant defenses, expression of glutamate receptor subtypes, and the balance of necrotic versus apoptotic machinery activated in response to injury. Expression of glutamate receptor subunit 4 on OL lineage cells, for example, occurs earlier in the SD rat compared with the LE rat, and in LE rats, the period of highest white matter susceptibility (P6) coincided with the highest expression of glutamate receptors on preOL, which peaked at this time [Talos et al., 2006a]. Such extrinsic factors might include differences in regulation of global cerebral blood flow and microcirculation via the neurovascular unit as well as differences in mobilization/activation of microglia and astrocytes in response to initial injury.

Given the wide variety of distinctly different rat models of WMI that have been developed in different strains of rat, direct comparison between models is currently dif- 
ficult. These models include perinatal exposure to hypoxia-ischemia, hypoxia, hyperoxia, endotoxin, cytotoxic cytokines, glutamate receptor agonists, malnutrition, toxins and other insults related to intrauterine growth retardation [Hagberg et al., 2002]. Comparison of these models would be facilitated by more standardized approaches to the neuropathological analysis of WMI. During the early phase of injury, one neuropathological index for comparison between strains and models is preOL degeneration. PreOL appear to be the major cell type that degenerates acutely in white matter in response to hypoxia-ischemia and lipopolysaccharide [Back et al., 2002; Lehnardt et al., 2002; Cai et al., 2003], but this notion may require revision as more sensitive injury markers become available for axons and other glial cell types.

During the delayed phase of injury, preOL display a robust regenerative response that could also serve as a useful endpoint for comparison between strains. Even with massive acute preOL degeneration, both OL progenitors and preOL increase in density severalfold within days after a hypoxic-ischemic insult in the rat [Segovia et al., 2008]. During this delayed phase of injury, preOL but not OL progenitors - undergo delayed apoptosis. Despite this ongoing preOL apoptosis, there is a net increase in total preOL due to a state of maturation arrest where preOL fail to differentiate and initiate myelination despite intact axons.

In order to comprehensively compare mechanisms of myelination failure between rat strains or models, analysis should include quantification of preOL and the extent of astrogliosis and visualization of the population of axons potentially available for myelination. The latter is important because extensive neuronal injury to rat cortical and subcortical gray matter structures may be accompanied by axon dysfunction and secondary myelination failure that is distinct from the myelination disturbances in human periventricular WMI. Current human neuropathological studies do not support that myelination failure in cerebral white matter primarily arises from neuronal degeneration in cortical or subcortical gray matter structures. However, WMI in preterm human survivors appears to be accompanied by a variable degree of neuronal loss or dysfunction, which is suspected to contribute to the cognitive and learning disabilities that are extremely common in school-age children diagnosed with the encephalopathy of prematurity [Volpe, 2009].

Regardless of the strain of rat analyzed, white matter maturation occurred rapidly by $\mathrm{P} 5-\mathrm{P} 7$, such that immature OL predominated. Thus, if WMI is sustained later in the first developmental week, it is likely to occur by mech- anisms distinct from that at $\mathrm{P} 2$, when preOL are the primary susceptible cell type in white matter, as in human cerebral white matter in the premature infant [Back et al., 2001]. In the SD rat at P7, a reduction in WMI coincided with greater resistance of immature OL to cell death from hypoxia-ischemia [Back et al., 2002]. In fact, both OL and early myelin sheaths stained more intensely for immature OL markers, which is consistent with a reactive oligodendroglial response. If disturbances in myelination are observed in response to injury during the immature OLpredominant window, this may be related to delayed immature OL degeneration, possibly in concert with failure of OL progenitors to generate a new preOL pool within lesions with chronic astrogliosis. However, such a mechanism has not been reported to date. Alternatively, it may be related to secondary neuroaxonal degeneration, which is commonly observed, for example, in response to hypoxia-ischemia at P7 [Vannucci et al., 1999].

\section{Clinical Correlations}

Given the heterogeneity in human cerebral maturation, there is a broad potential window when human white matter may be susceptible to injury. Epidemiological, neuropathological and neuroradiological studies support the highest incidence of preterm cerebral WMI occurs between about 23 weeks and 32 weeks gestation. Lesions have a particular predilection for frontal and parietal periventricular white matter, which appears related to the presence of a high density of preOL and a relatively lower density and proportion of immature OL and myelin [Back et al., 2001; Riddle et al., 2006; Talos et al., 2006b]. As early as P2, all three strains of rat had pretermequivalent features of OL lineage maturation that were similar to human cerebral white matter during the highrisk period for WMI. Moreover, white matter matures rapidly by $\mathrm{P} 5-\mathrm{P} 7$, such that the relative proportions of preOL and immature OL reverse with predominance of the latter. Our data are thus consistent with those from prior studies that support the near-term equivalence of the rat cerebrum by P7 [Vannucci et al., 1999].

There is little information regarding the timing of $\mathrm{OL}$ lineage maturation in cerebral gray matter in the neonatal rat relative to human in the first year of life. Although preOL are the major OL lineage stage present in preterm human parietal cortex [Back et al., 2001], maturation of the OL lineage in human cerebral cortex at term or later has not been systematically studied. Our study supports that maturation of the OL lineage occurred more slowly in unmyelinated regions of the cortex relative to subjacent white matter. Similarly, all three strains of rat 
showed a less mature pattern of OL lineage maturation in P14 cortex relative to adjacent white matter. This persistence of cortical preOL suggests that these cells may be vulnerable to hypoxic-ischemic lesions at P7. In fact, in the SD rat, significant acute degeneration of preOL occurred in the cerebral cortex despite a decline in white matter vulnerability at P7. WMI in preterm human survivors appears to be accompanied by a variable degree of delayed neuronal loss or dysfunction, which is suspected to contribute to the cognitive and learning disabilities that are extremely common in school-age children diagnosed with the encephalopathy of prematurity [Volpe, 2009]. Future studies are needed to determine whether preOL degeneration in human cerebral gray matter occurs and contributes to long-term cerebral dysfunction.

\section{Acknowledgments}

This study was supported by the National Institutes of Health grants 1RO1NS054044 (S.A.B.), 2R37NS045737-05 (S.A.B.), R01 NS31718 (F.E.J.) and P01 NS38475 (F.E.J. and J.J.V.), a Mental Retardation Developmental Disorders Research Center Grant (P30 HD18655) to Children's Hospital Boston, as well as the American Heart Association (Bugher Award) and the March of Dimes Birth Defects Foundation (S.A.B.).

\section{References}

Back SA (2006): Perinatal white matter injury: the changing spectrum of pathology and emerging insights into pathogenetic mechanisms. Ment Retard Dev Disabil Res Rev 12: 129-140.

- Back SA, Han BH, Luo NL, Chricton CA, Tam J, Xanthoudakis S, Arvin KL, Holtzman DM (2002): Selective vulnerability of late oligodendrocyte progenitors to hypoxia-ischemia. J Neurosci 22:455-463.

Back SA, Luo NL, Borenstein NS, Levine JM, Volpe JJ, Kinney HC (2001): Late oligodendrocyte progenitors coincide with the developmental window of vulnerability for human perinatal white matter injury. J Neurosci 21:1302-1312.

- Back SA, Luo NL, Mallinson RA, O’Malley JP, Wallen LD, Frei B, Morrow JD, Petito CK, Roberts JCT, Murdoch GH, Montine TJ (2005): Selective vulnerability of preterm white matter to oxidative damage defined by $\mathrm{F}_{2}$-isoprostanes. Ann Neurol 58:108-120.

- Back SA, Riddle A, Hohimer AR (2006): Role of instrumented fetal sheep preparations in defining the pathogenesis of human periventricular white matter injury. J Child Neurol 21:582-589.

Cai Z, Pang Y, Lin S, Rhodes P (2003): Differential roles of tumor necrosis factor- $\alpha$ and interleukin- $1 \beta$ in lipopolysaccharide-induced brain injury in the neonatal rat. Brain Res 975:37-47.

-Fan L, Mitchell H, Tien L, Zheng B, Pang Y, Rhodes P, Cai Z (2008): $\alpha$-Phenyl- $n$-tert-butyl-nitrone reduces lipopolysaccharide-induced white matter injury in the neonatal rat brain. Dev Neurobiol 68:365-378.

- Follett PL, Deng WD, Dai W, Talos D, Massillon L, Rosenberg PA, Volpe JJ, Jensen FE (2004): Glutamate receptor mediated oligodendrocyte toxicity in periventricular leukomalacia: a protective role for topiramate. J Neurosci 24:4412-4420.
Fontaine R, Olivier P, Massonneau V, Leroux P, Degos V, Lebon S, El Ghouzzi V, Lelièvre V, Gressens P, Baud O (2008): Vulnerability of white matter towards antenatal hypoxia is linked to a species-dependent regulation of glutamate receptor subunits. Proc Natl Acad Sci USA 105:16779-16784.

Hagberg H, Peebles D, Mallard C (2002): Models of white matter injury: comparison of infectious, hypoxic-ischemic, and excitotoxic insults. Ment Retard Dev Disabil Res Rev 8: 30-38.

- Haynes RL, Folkerth RD, Keefe RJ, Sung I, Swzeda LI, Rosenberg PA, Volpe JJ, Kinney HC (2003): Nitrosative and oxidative injury to premyelinating oligodendrocytes in periventricular leukomalacia. J Neuropathol Exp Neurol 62:441-450.

Jakovcevski I, Zecevic N (2005): Sequence of oligodendrocyte development in the human fetal telencephalon. Glia 49:480-491.

Jensen FE (2005): The role of glutamate receptors in periventricular leukomalacia. J Child Neurol 20:950-959.

Kinney HC, Armstrong DD (2002): Perinatal neuropathology; in Graham D, Lantos P (eds): Greenfield's Neuropathology, ed 7. London, Arnold, pp 519-606.

Lehnardt S, Lachance C, Patrizi S, Lefebvre S, Follett PL, Jensen FE, Rosenberg PA, Volpe JJ, Vartanian T (2002): The Toll-like receptor TLR4 is necessary for lipopolysaccharideinduced oligodendrocyte injury in the CNS. J Neurosci 22:2478-2486.

Manning S, Talos D, Zhou C, Selip D, Park H, Park C, Volpe J, Jensen F (2008): NMDA receptor blockade with memantine attenuates white matter injury in a rat model of periventricular leukomalacia. J Neurosci 25:66706678.
Riddle A, Luo N, Manese M, Beardsley D, Green L, Rorvik D, Kelly K, Barlow C, Kelly J, Hohimer A, Back S (2006): Spatial heterogeneity in oligodendrocyte lineage maturation and not cerebral blood flow predicts fetal ovine periventricular white matter injury. J Neurosci 26:3045-3055.

Segovia K, McClure M, Moravec M, Luo N, Wang Y, Gong X, Riddle A, Craig A, Struve J, Sherman L, Back S (2008): Arrested oligodendrocyte lineage maturation in chronic perinatal white matter injury. Ann Neurol 63:520-530.

Talos D, Fishman R, Park H, Folkerth R, Follett P, Volpe J, Jensen F (2006a): Developmental regulation of $\alpha$-amino-3-hydroxy-5-methyl4-isoxazole-propionic acid receptor subunit expression in forebrain and relationship to regional susceptibility to hypoxic/ischemic injury. I. Rodent cerebral white matter and cortex. J Comp Neurol 497:42-60.

Talos DM, Follett PL, Levada R, Folkerth R, Volpe JJ, Jensen FE (2006b): Developmental regulation of AMPA receptor subunit expression in forebrain and relationship to regional susceptibility to hypoxic/ischemic injury. Part II. Human cerebral cortex and white matter. J Comp Neurol 497:61-77.

- Vannucci RC, Connor JR, Mauger DT, Palmer C, Smith MB, Towfighi J, Vannucci SJ (1999): Rat model of perinatal hypoxic-ischemic brain damage. J Neurosci Res 55:158-163.

Volpe JJ (2008): Postnatal sepsis, necrotizing entercolitis [sic!], and the critical role of systemic inflammation in white matter injury in premature infants. J Pediatr 153:160-163.

-Volpe JJ (2009): Brain injury in premature infants: a complex amalgam of destructive and developmental disturbances. Lancet Neurol 8:110-124. 